\title{
Enhanced photon detection efficiency for next-generation neutrino telescopes using photon traps
}

\author{
Koun Choi ${ }^{a, b, *}$ and Carsten Rott $^{c, b}$ \\ ${ }^{a}$ Institute for Basic Science, \\ 55, Expo-ro, Yuseong-gu, Daejeon-si, South Korea \\ ${ }^{b}$ Sungkyunkwan University, Department of Physics, \\ 2066, Seobu-ro, Jangan-gu, Suwon-si, Gyeong Gi-do, South Korea \\ ${ }^{c}$ University of Utah, Department of Physics and Astronomy, \\ 201 Presidents' Cir, Salt Lake City, UT 84106, USA \\ E-mail: koun@ibs.re.kr, rott@physics.utah.edu
}

We propose a photon trap designed for improved photon detection efficiency in a cost-efficient way. Wavelength Shifting plastic sheets (WLS) are deployed at the bottom of a PMT, surrounded by dichroic film by which photons are efficiently trapped and guided to the PMT. We measured wave-length dependent transmittance of a commercially available dichroic film in water, a key variable determining photon trapping efficiency. We ran a Geant4 based simulation with the property of the commercially available dichroic film as a realistic case. We also ran a simulation with a hypothetical dichroic film whose bandpass is optimized to absorption and reemission spectra of the WLS and the quantum efficiency of the PMT, as an ideal case. The preliminary results of the photon collection and detection efficiency enhancements are computed, as well as timing distribution of the photons. We discuss how this new conceptual design can be applied to next-generation neutrino telescopes.

$37^{\text {th }}$ International Cosmic Ray Conference (ICRC 2021)

July 12th - 23rd, 2021

Online - Berlin, Germany

\footnotetext{
*Presenter
} 


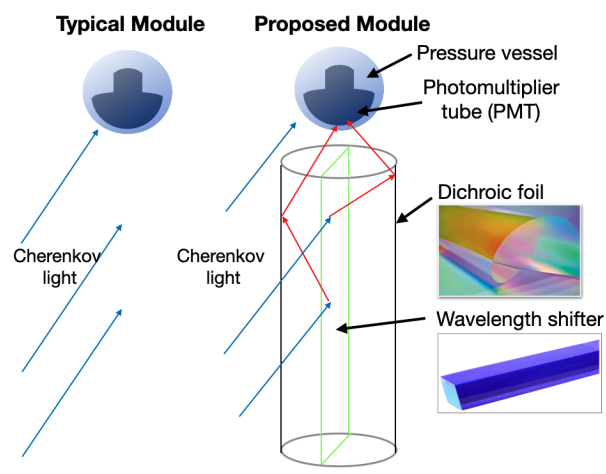

Figure 1: Basic design and concept of the proposed optical sensor module.

\section{Introduction}

The photon detection ability of large neutrino detectors and neutrino telescopes can be enhanced using photon traps that effectively channel light to their optical sensors such as photomultiplier tubes (PMTs). This detection principle has been proposed for Hyper-Kamiokande [1]. Here we discuss a conceptual design for neutrino telescopes, which are sparsely instrumented with compact optical sensors and hence offer opportunities to increase photo-sensitive area by channeling photons that propagate to their vicinity. A variety of concepts to achieve a similar goal has been discussed in the literature [2-4]. We propose a system that can be attached as an accessory to an existsing optical sensor to enhance its photon detection ability. Our goal is to develop a cost-effective optical module that relies on inexpensive materials and can be easily manufactured. For that, we propose a design to directly integrate the photosensitive materials in the detector medium that can create a very large photon trap at a substantially lower cost. Next generation instruments such as IceCube-Gen2 [5], KM3NeT [6], GVD [7] and P-ONE [8] can benefit from such design.

The design and detection principle of our optical module is shown in Fig. 1. Cherenkov light associated with neutrino interactions will efficiently pass the dichroic foil and hit a wavelength shifting (WLS) plates that absorbs and reemits photons at a different wavelength. Once photons are reemitted, they are reflected on the dichroic foil ('trapped'), and channeled to the PMTs, where they will be detected. Wavelength shifter and dichroic foil will be tuned in a way to maximize the trapping of photons and to match it to the peak quantum efficiency of the PMT. The design can be easily scaled and overcome module size limitations due to pressure vessels, for example.

\section{Measurement of the transmittance of dichroic film}

A dichroic film reflects photons in one or more spectral bands while transmitting others with nearly zero coefficient of absorption. Dichroic film is constructed from durable and flexible polyester materials and has a thickness of about $0.1 \mathrm{~mm}$. Dichroic film has been used for glass finishes and interior design, but has also been used for large-area photon detectors [9]. Materials used for outside applications typically come with a hard coated surface layer to resist scratching and to function as a UV filter. For the purpose of this study, we have considered two different dichroic films: (1) a 

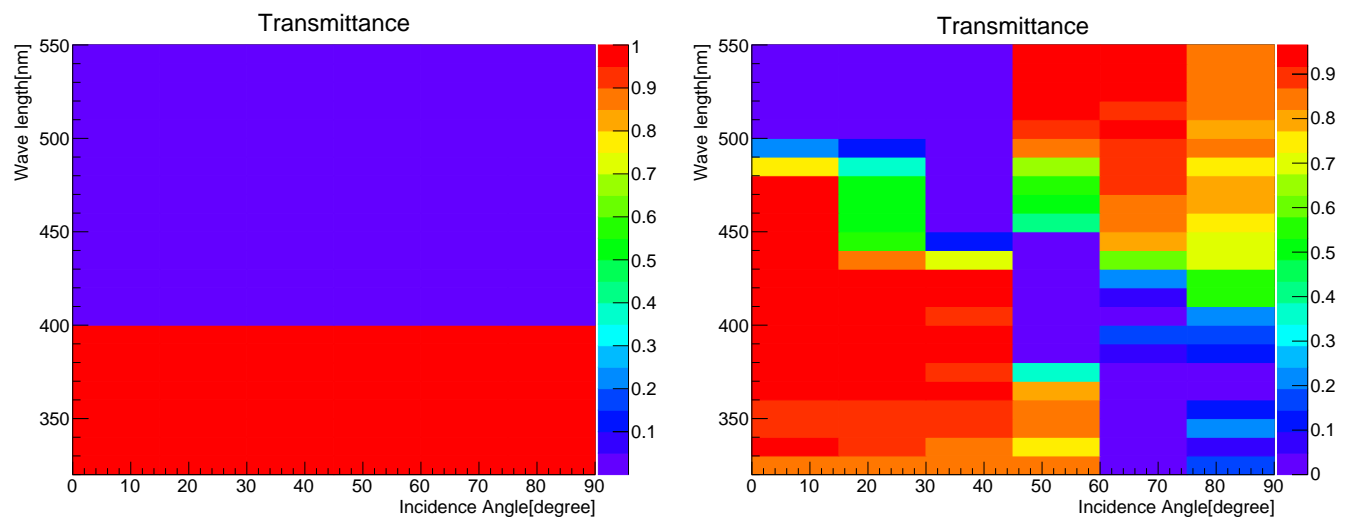

Figure 2: (left:) Transmittance of an ideal dichroic film, assumed to have 100\% transmittance below $400 \mathrm{~nm}$ at all incident angle and 0\% transmittance above. (right:) Transmittance of the $3 M$ DF Chill film measured in the water for the photon wavelength range of $330-550 \mathrm{~nm}$, at five incident angles $\left(0,30,45,60,75^{\circ}\right)$.

"realistic" dichroic film with the optical properties of commercially available film in the market called DF Chill produced by $3 M$, (2) an "ideal" version of a dichroic film with tuned band gap of full transmittance below $400 \mathrm{~nm}$ and zero transmittance above (see Fig.2 (left)), optimized to match the WLS emission spectrum and PMT quantum efficiency.

For the realistic case, we measured the transmittance of the DF Chill film in the water for various incident angles and wavelengths. Photons were injected from a Xenon lamp to a monochromator to generate monochromatic flux of $5 \mathrm{~nm}$ band width. Injected photons are detected with a photodiode after passing the dichroic film hanging inside the water tank. The measurement was performed for incident angles of $0,30,45,60^{\circ}$ and wavelengths of $330-550 \mathrm{~nm}$. The resultant transmittance is shown in Fig.2 (right). While the ideal film assumes to have a constant transmittance at all incident angles, in reality, the bandpass with maximum transmittance decreases as incident angle increases, which blocks primary photons. Also the product shows nonzero transmittance at high wavelength and high incident angle, resulting in ineffective photon trapping efficiency.

\section{Simulation study}

Using GEANT4 [10], we studied the photon detection efficiency of the proposed optical module. For this study, a 3-inch diameter half-sphere PMT with uniform photon angular acceptance is considered, with a quantum efficiency (QE) assumed to be identical with that of the Hamamatsu R12199 model [11].

At the bottom of the PMT, a cylindrical dichroic film with outside radius of $3.81 \mathrm{~cm}$ and height of $1 \mathrm{~m}$ is deployed, and two WLS plates which are perpendicularly crossed to each other are deployed inside the dichroic cylinder. The height, width and depth of the plates are $1.00 \mathrm{~m}, 5.62 \mathrm{~cm}$ and $1.00 \mathrm{~cm}$, respectively. The dichroic film extends to cover the entire PMT photocathode so the photons trapped inside the cylinder can be efficiently guided to the PMT.

EJ-286 from Eljen technology [12] is selected as wavelength shifting material for this study, for its broad absorption spectrum at $300-400 \mathrm{~nm}$ where Cherenkov photons are populated. The 

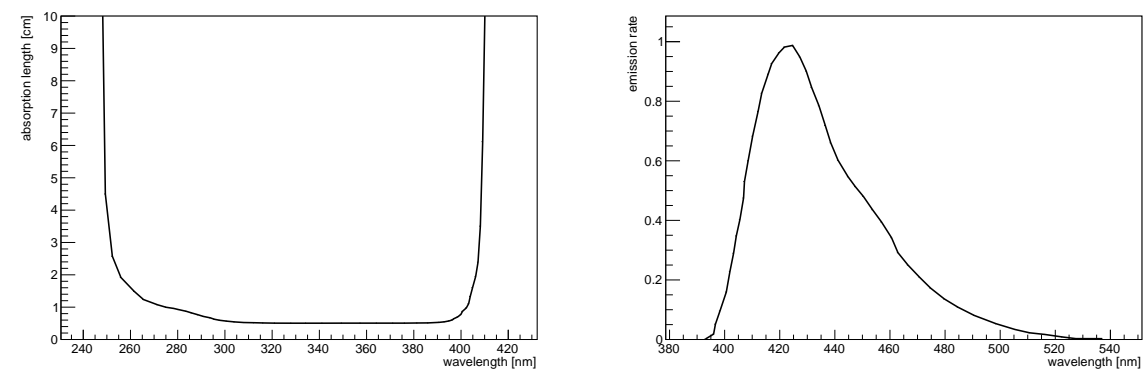

Figure 3: Absorption and reemission spectra of the EJ-286.

absorption spectrum is calculated for $5 \mathrm{~mm}$ thick plate as in the spec sheet provided by the producer, and extrapolated below $280 \mathrm{~nm}$. Figure 3 shows the absorption and reemission spectra of $E J$ 286. Its reemission spectrum raises above $400 \mathrm{~nm}$, showing marginal overlapping with absorption spectrum and optimally matching with the ideal dichroic film. In comparison to EJ-280 from Eljen technology or BC-482A from Saint-Gobain Crystals which reemit photons at higher wavelength, $E J-286$ matched better with the ideal dichroic film. The reemission rate of the absorbed photons at the peak is $92 \%$ and the refraction index is $\sim 1.59$, trapping photons with incident angle $>59^{\circ}$ within the plate by total internal reflection. An efficient dichroic cylinder may trap the rest of the photons with its good reflectance at low incident angle. The timing constant of the EJ-286 is $1.2 \mathrm{~ns}$.

For each tested set up, 10,000,000 photons are generated following $1 / \lambda^{2}$ distribution where $\lambda$ is the wavelength of a photon, as expected for Cherenkov radiation. The photons are injected at $(-2 \mathrm{~m}, 0, z)$ for the range of $z$ covering the height of the photon trap. The photons are injected uniformly in $\cos \theta=[-1,1]$. Mie scattering in the water may reduce number of primary photons and is turned off in the study. Water properties such as absorption length and refractive index are taken from the SKDETSIM simulation [13].

\section{Results}

As the ideal dichroic film reflects injected photons with wavelength longer than $400 \mathrm{~nm}$, number of arrived photons at the photo sensor (photon collection efficiency) decreases to $79 \%$ compared to PMT-only case (see Tab. 1). Number of photons detected by the PMT (photon detection efficiency), which is product of the photon collection efficiency and the QE as a function of $\lambda$, decreases to $71 \%$ compared to a PMT-only set up (7.1\% compared to $10 \%)$. In Table 1, Secondary collected/detected photons indicate the photons that are reemitted in the WLS plates and arrive at/detected by the PMT, respectively. The total number of detected primary and secondary photons with the ideal photon trap is $\sim 16$ times of the photons detected in the PMT-only set up (157\% compared to $10 \%$ ). Simulated result from photon trap with WLS plates without a dichroic film ("no mirror") is also shown for a comparison. In the realistic case with $3 \mathrm{M}$ film, there is $\sim 40 \%$ increase in total number of photons.

Photon collection and detection efficiency for various photon trap height is shown in Tab. 2 . For a photon trap with $8 \mathrm{~m}$ height, number of detected photons is about 77 times of the case without 


\begin{tabular}{|c|c|c|}
\hline & primary photons & secondary photons \\
\hline PMT only $(\times \mathrm{QE})$ & $1(0.10)$ & $0(0)$ \\
\hline ideal $(\times \mathrm{QE})$ & $0.79(0.071)$ & $6.1(1.5)$ \\
\hline $3 \mathrm{M}(\times \mathrm{QE})$ & $0.49(0.064)$ & $0.33(0.081 \pm 0.004)$ \\
\hline WLS only $(\times \mathrm{QE})$ & $0.94(0.097)$ & $0.51(0.12)$ \\
\hline
\end{tabular}

Table 1: Number of collected photons/detected photons (collected photons $\times$ QE) directly by the PMT (primary) and through the photon trap (secondary), for a PMT-only design (first row), photon trap design with ideal dichroic film(second row), photon trap with 3M commercial film(fourth row) and photon trap without a dichroic cylinder (fifth row). All numbers are divided by the collected primary photons by the PMT-only design (shown in the first row).

photon trap.

\begin{tabular}{|c|c|c|}
\hline & primary photons & secondary photons \\
\hline PMT $(\times \mathrm{QE})$ & $1(0.10)$ & $0(0)$ \\
\hline ideal $1 \mathrm{~m}(\times \mathrm{QE})$ & $0.79(0.071)$ & $6.1(1.5)$ \\
\hline ideal $2 \mathrm{~m}(\times \mathrm{QE})$ & $0.79(0.072)$ & $12(2.7)$ \\
\hline ideal $4 \mathrm{~m}(\times \mathrm{QE})$ & $0.77(0.067)$ & $21(4.9)$ \\
\hline ideal $8 \mathrm{~m}(\times \mathrm{QE})$ & $0.76(0.070)$ & $34(7.6)$ \\
\hline
\end{tabular}

Table 2: Number of collected photons/detected photons (collected photons $\times$ QE) directly by the PMT (primary) and through the photon trap (secondary), for a PMT-only design (first row), photon trap design with ideal dichroic film for various heights ( 2 - 5th rows). All numbers are divided by the collected primary photons by the PMT-only design (shown in the first row).

Figure 4 (top left) shows secondary photon collection efficiency as a function of absorption position $z$. secondary Photons initiated close to the photo sensor has larger chance to hit the photo sensor, however time spent while bouncing multiple times on the photon trap wall before arrival dominates. Figure 4 (bottom left) shows arrival timing distribution of primary (black) and secondary photons on the PMT photocathode for ideal case for $1 \mathrm{~m}$ height (red solid). The secondary photons have longer timing distribution; the mean of the delayed time (secondary photon arrival time - mean of the primary photon arrival time) is $17.5 \mathrm{~ns}$. The RMS of the secondary photon timing distribution is $23 \mathrm{~ns}$, where that of the primary photons is $6.9 \mathrm{~ns}$. Arrival timing distribution of ideal case for $8 \mathrm{~m}$-long photon trap is also shown in red dashed line. The arrival time increases for longer photon trap design, as photons experience more bounces before reaching the PMT. This is seen better in Fig. 4 (top left), where the arrival timing distribution of secondary photons in ideal case is shown as a function of the absorption position $z$, for four different photon trap height. Figure 4 (bottom right) shows cumulative arrival timing distributions. 

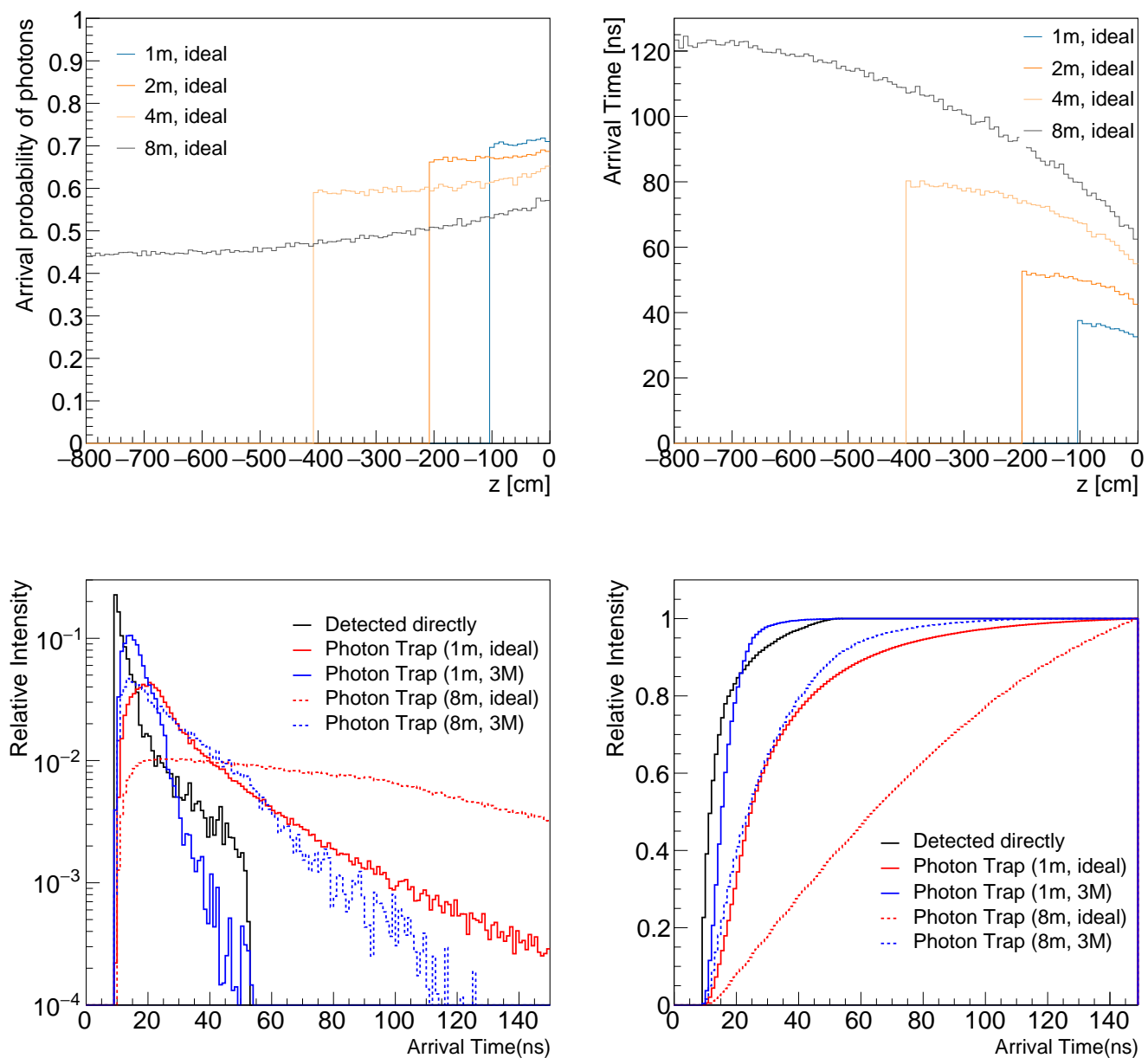

Figure 4: (top left:) Arrival probability of secondary photons as a function of initial absorption position $z$, for ideal photon traps with height of 1,2,4,8m. (top right:) Arrival time of secondary photons on the PMT photocathode as a function of initial photon absorption position $z$, for 4 different photon trap heights in ideal configuration. (bottom left:) Normalized averaged arrival time of primary (black) and secondary photons on the PMT photocathode (relative y-axis scale is arbitrary). (bottom right:) Accumulated arrival time of primary (black) and secondary photons on the PMT photocathode (normalized to 1).

\section{Conclusion}

Photon traps could be used for better calorimetry and vetoing in future neutrino telescopes. In this work, a simple and cost-effective design has been proposed and promising preliminary result was derived for an idealized set up. Running a simulation with a dichroic film with ideal band pass and perfect mirroring, we obtained a sixteen times enhanced photon detection efficiency for $1 \mathrm{~m}$-height photon trap.

Feasibility of the design requires further studies and the design has a room to be improved. In near future, we will pursue the study by checking the possibility to optimize the band pass of the dichroic film. Current study matches diameter of the trap with PMT size, but a photon trap 
could potentially be larger and photons could be channeled to the PMT via light guide.Note that the current design ignores cables, holding structures, single WLS plate, etc. that also needs a detailed discussion before making a prototype detector, as we as discussion on supporting structures for dichroic film and WLS plates.

In case of multi-PMT modules, our proposed system could be attached to one or more PMTs of such a module. As an example for IceCube-Gen2, the bottom or top PMT of multi-PMT modules that are aligned with the drill hole can be dedicated to photon trap to serve for calorimetric measurement, while other PMTs provide good timing information. One can also achieve directional photon sensitivity through segmentation of the proposed model, thereby improving over existing modules in another way.

\section{References}

[1] C. Rott, S. In, F. Retière and P. Gumplinger, JINST 12 (2017) no.11, P11021 doi:10.1088/17480221/12/11/P11021 [arXiv:1708.01702 [astro-ph.IM]].

[2] I. Bartos, Z. Marka and S. Marka, Nature Commun. 9 (2018) no.1, 1236 doi:10.1038/s41467018-03693-z [arXiv:1706.06589 [physics.ins-det]].

[3] M. Ehlert, A. Hollnagel, I. Korol, A. Korzenev, H. Lacker, P. Mermod, J. Schliwinski, L. Shihora, P. Venkova and M. Wurm, JINST 14 (2019) no.03, P03021 doi:10.1088/17480221/14/03/P03021 [arXiv:1812.06460 [physics.ins-det]].

[4] J. Rack-Helleis et al. [IceCube], PoS ICRC2021 (2021), 1038 [arXiv:2107.10194 [astroph.HE]].

[5] M. G. Aartsen et al. [IceCube-Gen2], J. Phys. G 48 (2021) no.6, 060501 doi:10.1088/13616471/abbd48 [arXiv:2008.04323 [astro-ph.HE]]

[6] S. Adrian-Martinez et al. [KM3Net], J. Phys. G 43 (2016) no.8, 084001 doi:10.1088/09543899/43/8/084001 [arXiv:1601.07459 [astro-ph.IM]].

[7] A. D. Avrorin, A. V. Avrorin, V. M. Aynutdinov, R. Bannasch, I. A. Belolaptikov, D. Y. Bogorodsky, V. B. Brudanin, N. M. Budnev, I. A. Danilchenko and S. V. Demidov, et al. JETP Lett. 101 (2015) no.5, 289-294 doi:10.1134/S0021364015050021 [arXiv:1412.3672 [astroph.HE]].

[8] M. Agostini et al. [P-ONE], Nature Astron. 4 (2020) no.10, 913-915 doi:10.1038/s41550-0201182-4 [arXiv:2005.09493 [astro-ph.HE]].

[9] M. Auger, Y. Chen, A. Ereditato, D. Goeldi, I. Kreslo, D. Lorca, M. Luethi, T. Mettler, J. R. Sinclair and M. S. Weber, Instruments 2 (2018) no.1, 3 doi:10.3390/instruments2010003 [arXiv:1711.11409 [physics.ins-det]].

[10] S. Agostinelli et al. [GEANT4], Nucl. Instrum. Meth. A 506 (2003), 250-303 doi:10.1016/S0168-9002(03)01368-8 
[11] https://wWw.hamamatsu.com/resources/pdf/etd/p-dev_2020_T0TH0029E.pdf

[12] https://eljentechnology.com/products/wavelength-shifting-plastics/ ej-280-ej-282-ej-284-ej-286

[13] K. Abe, Y. Hayato, T. Iida, K. Iyogi, J. Kameda, Y. Kishimoto, Y. Koshio, L. Marti, M. Miura and S. Moriyama, et al. Nucl. Instrum. Meth. A 737 (2014), 253-272 doi:10.1016/j.nima.2013.11.081 [arXiv:1307.0162 [physics.ins-det]]. 